\title{
FLUORIDE RELEASE FROM RMGIC VERSUS GIOMER CONCERNING DIFFERENT CURING DEVICES AT DIFFERENT TIME INTERVALS (AN IN-VITRO STUDY)
}

\author{
Ibrahim Barakat* and Ramy Abdallah Abdelrahim***
}

\begin{abstract}
This in-vitro study was directed to evaluate the effect of argon laser, visible lights, and lightemitting diode curing devices on fluoride release and fluoride recharge of RMGIC and Giomer.

Methodology: A total of 120 non-carious anterior primary teeth were included in this study. Teeth were divided into two equal main groups $(n=60)$ according to the type of resin restorative material. The first group; RMGIC restorative material, the second group; Giomer restorative material. Each main group was subdivided into three subgroups according to the light-curing unit; argon laser, light-emitting diode, and quartz tungsten halogen.

Results: The results of fluoride release and re-release revealed that RMGIC has significantly higher fluoride release when compared to Giomer restorative material at different time intervals and with different light-curing units. Moreover, the results of this study exhibited that the fluoride release and re-release decrease significantly in descending order over the time intervals of this study (24 hours, 1 week, 2 weeks, 3 weeks, and at one month) respectively. In addition, the results of the present study showed that the fluoride release and re-release decrease significantly in descending order with the use of argon laser, quartz tungsten halogen, and light-emitting diode respectively.

Conclusions: RMGIC has a higher fluoride release and recharging ability when compared to Giomer. The use of QTH light-curing unit resulted in higher fluoride release/re-release when compared to LED and argon laser LCUs.
\end{abstract}

KEYWORDS: Fluoride release, Fluoride recharge, RMGIC, and Giomer.

\section{INTRODUCTION}

Fluoride increases the tooth resistance to caries through different protective mechanisms such as inhibition of demineralization ability and enhances remineralization ability; moreover, it plays a significant role in the inhibition of microbial growth of cariogenic bacteria. ${ }^{(1)}$

* Assistant Professor, Department of Pedodontics and Oral Health, Faculty of Dental Medicine, Al-Azhar University, Cairo, Egypt.

** Associate Professor, Dental Biomaterials, Faculty of Dentistry, Al-Azhar university, Cairo, Egypt 
Fluoride-containing restorative materials can be recharged with fluoride by using fluoridated products including; toothpaste, and mouthwashes, in addition to acidulated phosphate fluoride (APF) gel. ${ }^{(2,3)}$ This recharging ability may contribute to their long-term effectiveness in caries inhibition.

Amongst the fluoride-releasing restorative materials, conventional glass ionomer cement (GIC) exhibit the high efficacy in resisting secondary caries formation around restorations. ${ }^{(4)}$ However, they are inferior in comparison to composite resins because of high moisture sensitivity, low initial mechanical properties, and inferior translucency. ${ }^{(5)}$

To overcome the shortcomings of GICs while maintaining their clinical advantage in caries inhibition, hybrid materials that purportedly combine the benefits of glass ionomers and composite resins were developed. ${ }^{(6)}$ Examples include resin-modified glass ionomer cement (RMGIC), polyacid-modified composite resins (compomers), and giomer.

The curing of resin-based restorative materials with light-curing units is considered an integral part of modern dentistry. There are different light-curing units (LCUs) in the dental clinic such as; halogenbased LCUs, and light-emitting diode (LED). ${ }^{(7)}$ More recently, argon laser has been approved for initiating the setting reaction with visible light cured resins. ${ }^{(8)}$

However, adequate polymerization efficiency of these curing units is a crucial factor in obtaining optimal clinical performance of composite resin restorative materials. ${ }^{(9,10)}$ Inadequate polymerization of resin-based restorative materials can associate with several problems such as; inferior physical properties, solubility in the oral environment, and increased microleakage with resultant recurrent decay and pulpal irritation. ${ }^{(11)}$

Although authors have focused on the effect of fluoride-containing restorative materials on fluoride release/recharge, and microleakage. While relatively, few studies examined the effect of curing units on fluoride release/recharge of these restorative materials. Therefore, this in vitro study investigates the effect of argon laser, halogen-based visible lights, and light-emitting diode curing systems on the fluoride release and recharge from two fluoridecontaining resin restorative materials.

\section{Aim of the study:}

This in vitro study was directed to evaluate the effect of argon laser, visible lights, and light-emitting diode curing devices on fluoride release and fluoride recharge of two fluoride-containing resin restorative materials namely RMGIC and Giomer.

\section{MATERIALS AND METHODS}

This study was carried out after approval of the ethical committee, Faculty of Dental Medicine, Al-Azhar University, Boys, Cairo (EC Ref No. $572 / 3259)$.

\section{Sample Size:}

The sample size was calculated based upon the results of Dionysopoulos et al ${ }^{(4)}$. The power test for sample size showed that; the effect size $(\mathrm{dz}=1.957)$ and the required sample size were calculated for $\mathrm{a}=0.05$ and a power of 0.95 , assuming a normal distribution. For this study, a sample size of 10 was obtained.

A total of 120 non-carious anterior primary teeth were used in this study and the teeth were divided into two equal main groups $(n=60)$ according to the type of resin restorative material. In each main group, each prepared cavity was restored with its assigned restorative material according to the manufacturer's instructions.

\section{Groups:}

- Group A: Restored with a resin-modified glass ionomer (Riva)*.

\footnotetext{
* Riva light cure HV Capsule, SDI Dental limited Victoria, Australia.
} 
- Group B: Restored with Giomer* (Beautiful flow plus).

Then, the samples of each main group were then subdivided into three equal subgroups $(n=20)$ according to the type of the curing system.

- Subgroup 1: Samples cured with a QTH lightcuring system ${ }^{* *}$ for 40 seconds (control group).

- Subgroup 2: Samples cured with an LED curing system ${ }^{* * * *}$ for 20 seconds.

- Subgroup 3: Samples cured with Argon laser curing system ${ }^{* * * *}$ for $10 \mathrm{sec}$. with output energy of $350 \mathrm{mj}$ frequency of $10 \mathrm{~Hz}$, power of $3.5 \mathrm{w}$, the wavelength of $2.94 \mu \mathrm{m}$, and short pulse mode of $230 \mu$ s).

Then, each subgroup was further categorized into two categories ( $\mathrm{a}$ and $\mathrm{b})(\mathrm{n}=10)$ according to the test (fluoride release/recharge)

The teeth used in this study were; non-carious anterior primary teeth extracted due to trauma or normally exfoliated, free from cracks, or any developmental defects. Surfaces of all teeth included in the study were cleaned from debris and blood using fluoride-free pumice and a lowspeed handpiece. Teeth were stored in normal saline until use. ${ }^{(1,14)}$ Standard non-beveled buccal class $\mathrm{V}$ cavities were prepared following the cavity preparation guidelines for composite ( $3 \mathrm{~mm}$ wide, 2 $\mathrm{mm}$ long, and $1 \mathrm{~mm}$ deep) in the cervical $1 / 3$ of each tooth using a standard \# 330 diamond bur mounted at high speed with air/water-cooled handpiece. ${ }^{(1)}$ The bur's length and a millimeter ruler were used to measure the dimensions of the cavity. ${ }^{(12)}$ All prepared teeth were thoroughly cleaned with water and gently dried before the application of each restorative material (figure 1).

\section{Artificial Saliva Preparation ${ }^{(13)}$ :}

Artificial saliva was prepared by adding $0.400 \mathrm{~g}$ natrium chloride, $0.400 \mathrm{~g}$ potassium chloride, $0.795 \mathrm{~g}$ calcium chloride monohydrate, $0.69 \mathrm{~g}$ sodium dihydrogen phosphate, $0.005 \mathrm{~g}$ sodium sulfide non-anhydrate and $1.0 \mathrm{~g}$ urea to $1000 \mathrm{~mL}$ distilled water. $\mathrm{pH}$ was adjusted to seven. Containers were incubated and stored at $37^{\circ} \mathrm{C}$ for $24 \mathrm{~h}$.

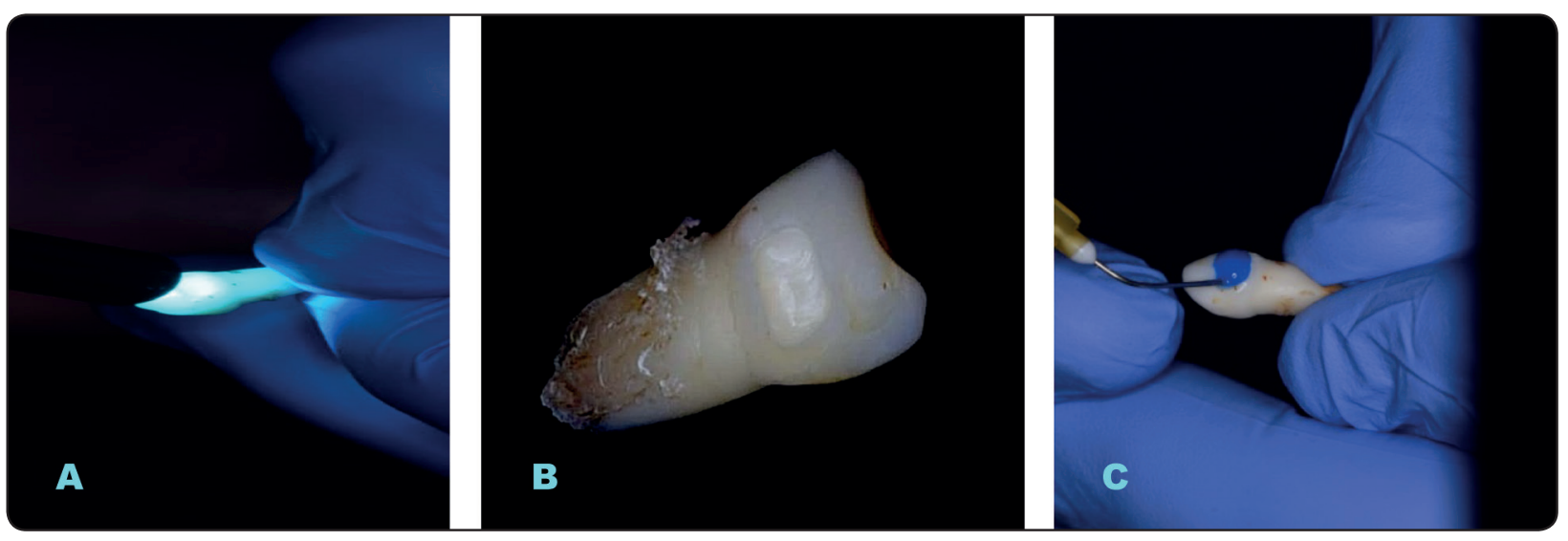

Fig. (1): (A) cavity preparation, (B) acid etch and (C) restoration curing

\footnotetext{
* Shofu Dental GmbH, Kyoto, Japan

** COXO-DB-682, DeepBlue Technology, Co., Limited, China.

*** COXO-DB-682, DeepBlue Technology, Co., Limited, China.

**** Smart 2940 plus, Deka, Italy.
} 


\section{Evaluation of fluoride release:}

After curing the restoration in each group, each tooth was transferred individually in plastic containers containing 4-ml of artificial saliva. After 24 hours incubation, the plastic containers were thoroughly shaken and the first fluoride concentration measurement of each specimen was performed. Then, teeth were removed, washed with 4-ml of distilled water, and transferred to a new container, containing 4-ml of fresh artificial saliva. Cumulative fluoride concentration in artificial saliva was measured on the 1st, 7th, 14th, 21st, and 30th days. ${ }^{(4,13)}$ Fluoride concentration was measured using a microanalytical technique* with an inverted fluoride ion-selective electrode. All measurements were per-

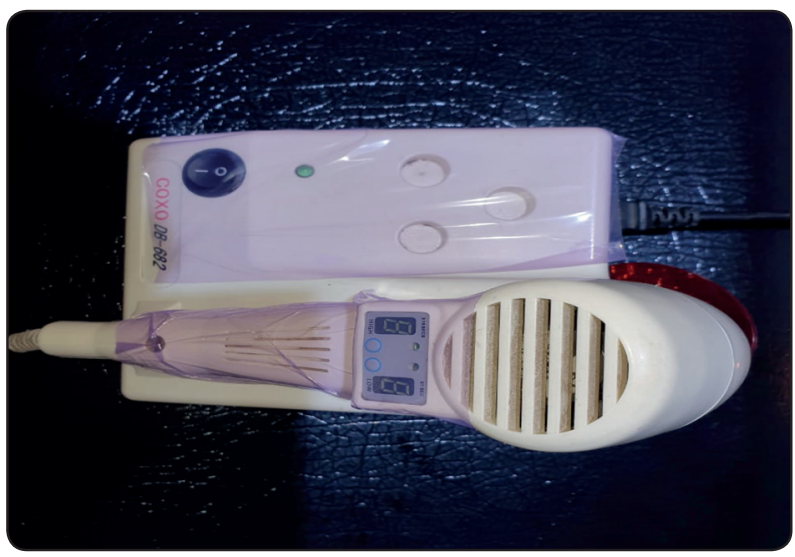

Fig. (2): QTH light curing unit.

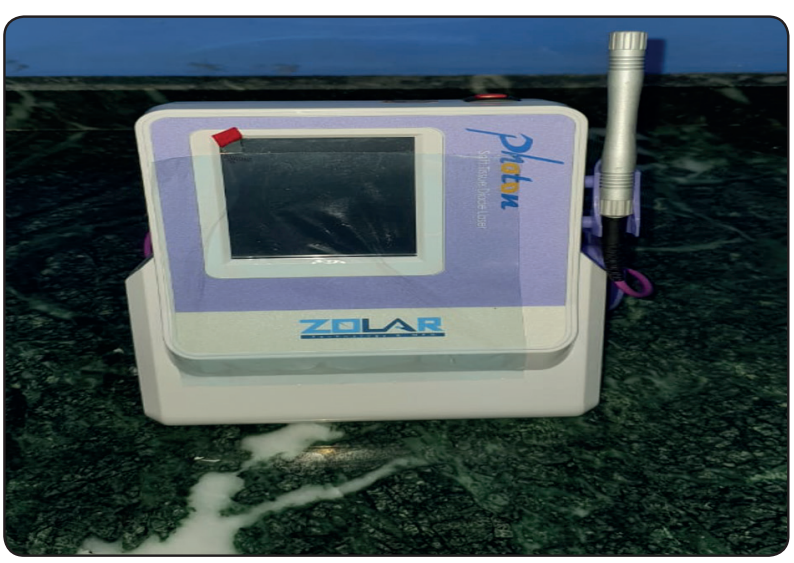

Fig. (4): Argon laser curing unit. formed at a constant room temperature of $23^{\circ} \mathrm{C}$ and were recorded as part per million (ppm) for statistical analysis. ${ }^{(4,13)}$ (Figure 5)

\section{Evaluation of fluoride recharging/re-release}

After 30 days of initial fluoride release, fluoridated gel was applied on the buccal surface of each tooth in each tested group, using a disposable brush, and allowed to dry for 5 minutes according to the manufacturer's instructions. Each tooth was stored in 4-ml of fresh artificial saliva with zero ppm fluoride ion concentration and was incubated at $37^{\circ} \mathrm{c}$ for 24 hours. Cumulative fluoride re-release was measured on the 1st, 7th, 14th, 21st, and 30th days. ${ }^{(4,13)}$

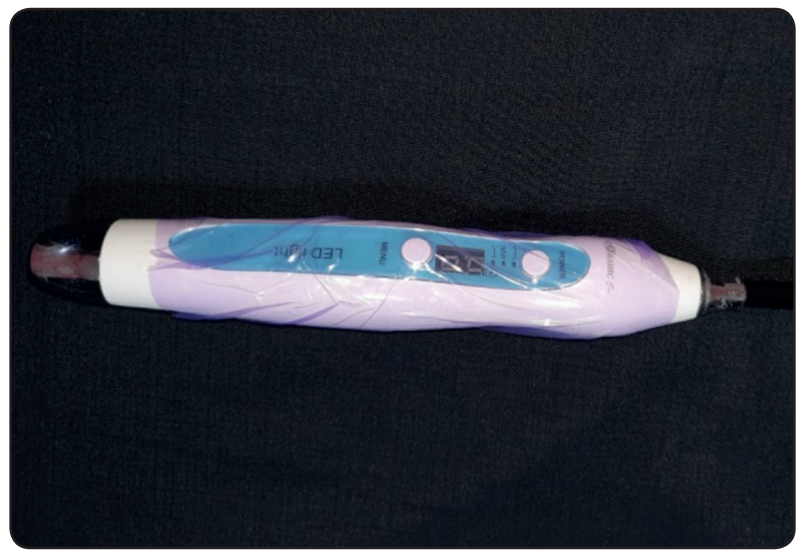

Fig. (3): LED light curing unit.

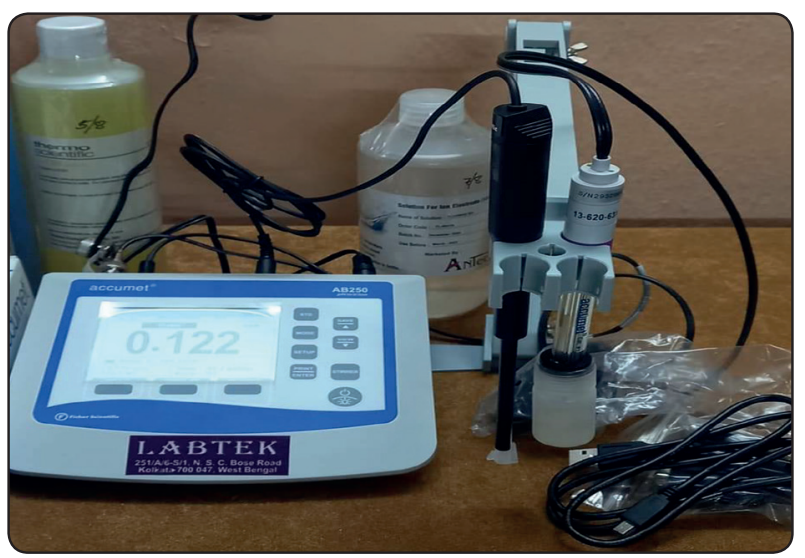

Fig. (5): Fluoride measuring.

\footnotetext{
* Ion Check 45, Radiometer analytical, France.
} 
Fluoride concentration was measured using a microanalytical technique as mentioned previously.

Data management and Statistical analysis: The collected data during study were tabulated and statistically analyzed using the ANOVA test; using SPSS version 22. The ANOVA level of significance was at $p$-value $<0$. Comparison among the groups was done using Post-Hock's test.

\section{RESULTS}

A- Comparison of fluoride release from RMGIC and Giomer with different curing devices at different time intervals:

The statistical analysis of fluoride release (ppm) of RMGIC cured with different curing devices at different time intervals revealed that; the difference in fluoride release was statistically significant as indicated by Two-way ANOVA test. There was statistically significant difference in fluoride release (ppm) of RMGIC when compared regarding to the curing device with significant level of $(p=0.00000)$.
Also, there were statistically significant difference in fluoride release (ppm) of RMGIC when compared regarding to the different time intervals with significant level of $(p=0.00000)$. The statistical analysis of fluoride release (ppm) of Giomer cured with different curing devices at different time intervals revealed that; the difference in fluoride release was statistically significant as indicated by Two-way ANOVA test. There was statistically significant difference in fluoride release (ppm) of Giomer when compared regarding to the curing device with significant level of $(p=0.00000)$. Also, there were statistically significant difference in fluoride release (ppm) of Giomer when compared regarding to the different time intervals with significant level of $(p=0.00000)$. Regarding to time storage intervals, there was statistically significant difference in amount fluoride release between Giomer and RMGIC. Also, regarding to the curing devices there was also a statistically significant difference in the amount of fluoride release. Where the RMGIC showed the higher amount of fluoride released when compared to Giomer as .showed in table (1).

TABLE (1): Comparison of fluoride release (ppm) from RMGIC and Giomer after curing with different curing systems at different time intervals:

\begin{tabular}{|c|c|c|c|c|c|c|c|c|c|}
\hline \multirow{2}{*}{ Variable } & \multicolumn{3}{|c|}{ QTH } & \multicolumn{3}{|c|}{ LED } & \multicolumn{3}{|c|}{ Argon laser } \\
\hline & RMGIC & Giomer & $p$-value & RMGIC & Giomer & $p$-value & RMGIC & Giomer & $p$-value \\
\hline $1^{\text {st }}$ day & $2.44 \pm 0.167^{\mathrm{Aa}}$ & $2.02 \pm 0.192^{\mathrm{Ab}}$ & $0.0000^{*}$ & $1.9 \pm 0.187^{\mathrm{Aa}}$ & $1.46 \pm 0.241^{\mathrm{Ab}}$ & $0.0000^{*}$ & $1.38 \pm 0.148^{\mathrm{Aa}}$ & $1.28 \pm 0.148^{\mathrm{Ab}}$ & $0.0000 *$ \\
\hline $7^{\text {th }}$ day & $2.06 \pm 0.152^{\mathrm{Ba}}$ & $1.5 \pm 0.158^{\mathrm{Bb}}$ & $0.0000 *$ & $1.48 \pm 0.148^{\mathrm{Ba}}$ & $1.08 \pm 0.239^{\mathrm{Bb}}$ & $0.0000^{*}$ & $1.06 \pm 0.114^{\mathrm{Ba}}$ & $0.92 \pm 0.130^{\mathrm{Bb}}$ & $0.0000 *$ \\
\hline $14^{\text {th }}$ day & $1.56 \pm 0.270^{\mathrm{Ca}}$ & $1.18 \pm 0.148^{\mathrm{Cb}}$ & $0.0000 *$ & $1.00 \pm 0.158^{\mathrm{Ca}}$ & $0.7 \pm 0.158^{\mathrm{cb}}$ & $0.0000^{*}$ & $0.7 \pm 0.158^{\mathrm{Ca}}$ & $0.62 \pm 0.130^{\mathrm{Cb}}$ & $0.0000 *$ \\
\hline $21^{\text {st }}$ day & $1.00 \pm 0.224^{\mathrm{Da}}$ & $0.76 \pm 0.114^{\mathrm{Db}}$ & $0.0000 *$ & $0.68 \pm 0.148^{\mathrm{Da}}$ & $0.28 \pm 0.130^{\mathrm{Db}}$ & $0.0000 *$ & $0.4 \pm 0.1^{\mathrm{Da}}$ & $0.24 \pm 0.055^{\mathrm{Db}}$ & $0.0000^{*}$ \\
\hline $30^{\text {th }}$ day & $0.44 \pm 0.114^{\mathrm{Ea}}$ & $0.42 \pm 0.084^{\mathrm{Eb}}$ & $0.0000 *$ & $0.28 \pm 0.084^{\mathrm{Ea}}$ & $0.046 \pm 0.011^{\mathrm{Db}}$ & $0.0000 *$ & $0.116 \pm 0.047^{\mathrm{Ea}}$ & $0.028 \pm 0.008^{\mathrm{Eb}}$ & $0.0000 *$ \\
\hline$p$-value & 0.00 & $00 *$ & & 0.00 & $00 *$ & & 0.00 & $11 *$ & \\
\hline
\end{tabular}

*; The results statistically at $p<0.05$. ; different capital litters in the same column indicted statistically significant.; different small litters in the same raw indicted statistically significant.; ns= non-significant. 
B- Comparison of fluoride re-release from RMGIC and Giomer with different curing devices at different time intervals:

The statistical analysis of fluoride re-release (ppm) of RMGIC cured with different curing devices at different time intervals revealed that; the difference in fluoride re-release was statistically significant as indicated by Two-way ANOVA test. There was statistically significant difference in fluoride re-release (ppm) of RMGIC when compared regarding to the curing device with significant level of $(p=0.00000)$. Also, there were statistically significant difference in fluoride rerelease (ppm) of RMGIC when compared regarding to the different time intervals with significant level of $(p=0.00000)$. The statistical analysis of fluoride re-release (ppm) of Giomer cured with different curing devices at different time intervals revealed that; the difference in fluoride re-release was statistically significant as indicated by Two-way ANOVA test. There was statistically significant difference in fluoride re-release (ppm) of Giomer when compared regarding to the curing device with significant level of $(p=0.00000)$. Also, there were statically significant difference in fluoride rerelease (ppm) of Giomer when compared regarding to the different time intervals with significant level of ( $p=0.00000)$. Regarding to time storage intervals, there was statistically significant difference in amount fluoride re-release between Giomer and RMGIC. Also, regarding to the curing devices there was also a statistically significant difference in the amount of fluoride release except for laser curing device as there was no significant difference. Where the RMGIC showed the higher amount of fluoride released when compared to Giomer as showed in table (2).

TABLE (2): Comparison of fluoride re-release (ppm) from RMGIC and Giomer after curing with different curing systems at different time intervals:

\begin{tabular}{|c|c|c|c|c|c|c|c|c|c|}
\hline \multirow{2}{*}{ Variable } & \multicolumn{3}{|c|}{ QTH } & \multicolumn{3}{|c|}{ LED } & \multicolumn{3}{|c|}{ Laser } \\
\hline & RMGIC & Giomer & $p$-value & RMGIC & Giomer & p-value & RMGIC & Giomer & $p$-value \\
\hline $1^{\text {st }}$ day & $1.74 \pm 0.114^{\mathrm{Aa}}$ & $1.48 \pm 0.084^{\mathrm{Ab}}$ & $0.0000 *$ & $1.48 \pm 0.148^{\mathrm{Aa}}$ & $1.08 \pm 0.084^{\mathrm{Ab}}$ & $0.0000 *$ & $1.08 \pm 0.084^{\mathrm{Aa}}$ & $1.00 \pm 0.071^{\mathrm{Ab}}$ & $0.0000^{*}$ \\
\hline $7^{\text {th }}$ day & $1.06 \pm 0.055^{\mathrm{Ba}}$ & $1.00 \pm 0.1^{\mathrm{Bb}}$ & $0.0000 *$ & $0.68 \pm 0.084^{\mathrm{Ba}}$ & $0.72 \pm 0.130^{\mathrm{Bb}}$ & $0.0000 *$ & $0.38 \pm 0.084^{\mathrm{Ba}}$ & $0.56 \pm 0.114^{\mathrm{Bb}}$ & $0.0000 *$ \\
\hline $14^{\text {th }}$ day & $0.78 \pm 0.084^{\mathrm{Ca}}$ & $0.58 \pm 0.084^{\mathrm{Cb}}$ & $0.0000 *$ & $0.38 \pm 0.084^{\mathrm{Ca}}$ & $0.4 \pm 0.071^{\mathrm{Cb}}$ & $0.0000 *$ & $0.16 \pm 0.055^{\mathrm{Ca}}$ & $0.18 \pm 0.084^{\mathrm{Cb}}$ & $0.0000 *$ \\
\hline $21^{\text {st }}$ day & $0.48 \pm 0.084^{\mathrm{Da}}$ & $0.32 \pm 0.084^{\mathrm{Db}}$ & $0.0000^{*}$ & $0.092 \pm 0.008^{\mathrm{Da}}$ & $0.094 \pm 0.009^{\mathrm{Db}}$ & $0.0000 *$ & $0.052 \pm 0.008^{\mathrm{CDa}}$ & $0.062 \pm 0.0084^{\mathrm{CDb}}$ & $0.0000^{*}$ \\
\hline $30^{\text {th }}$ day & $0.116 \pm 0.047^{\text {Еа }}$ & $0.086 \pm 0.011^{\mathrm{Eb}}$ & $0.0000^{*}$ & $0.062 \pm 0.008^{\mathrm{Da}}$ & $0.048 \pm 0.013^{\mathrm{Db}}$ & $0.0000 *$ & $0.014 \pm 0.005^{\mathrm{Da}}$ & $0.014 \pm 0.00^{5 \mathrm{D}} \mathrm{b}$ & $0.0000 *$ \\
\hline$p$-value & 0.00 & $00 *$ & & 0.0 & & & & $48 \mathrm{~ns}$ & \\
\hline
\end{tabular}

*; The results statistically at $p<0.05$.

; different capital litters in the same column indicted statistically significant.

; different small litters in the same raw indicted statistically significant.

; ns= non-significant. 


\section{DISCUSSION}

In the current study, RMGIC and Giomer were selected as alternative restorative materials to overcome the shortcomings of the conventional GICs while maintaining their clinical advantage in caries inhibition, as these hybrid materials purportedly combine the benefits of glass ionomers and composite resins. ${ }^{(6)}$

Artificial saliva with no fluoride content was used in the current study to avoid the presence of fluoride ions in natural saliva, which may act as a confounding factor, so that the fluoride amount obtained in this study was the pure amount of fluoride ion derived from RMGIC or Giomer fluoride release. ${ }^{(15,16)}$

Many methods have been employed to estimate the amount of fluoride releases such as spectrophotometry, ion chromatography, fluoride ion-specific electrodes, and capillary electrophoresis. ${ }^{(1)}$ Ion-specific electrode with an ion analyzer was used in this study because it is simple, inexpensive and does not require the use of complex laboratory equipment. Moreover, it gives an accurate and direct estimate of the free fluoride present in the solution. ${ }^{(17)}$

The results of the present study revealed that the amount of fluoride release, as well as fluoride re-release of the RMGIC and Giomer on the $1^{\text {st }}$ day, was higher among all tested periods under different curing methods. This may be attributed to the fluoride release and re-release on the $1^{\text {st }}$ day were induced by superficial rinsing effect and during the subsequent days release was attributed to its ability to diffuse through cement pores and fractures. ${ }^{(18)}$ Fluoride release can occur in response to water uptake after the dissolution of the glass filler particles or the ionic reaction on the surface of the glass particles. ${ }^{(19)}$

In addition, the first process of fluoride release from the surface of RMGIC and Giomer after which the elution is markedly reduced, accompanied by the second bulk diffusion process by which small amounts of fluoride continue to be released into the surrounding media. This pattern of release has been observed in previous studies. ${ }^{(16,20)}$ However, Giomer has no initial burst action as RMGIC but Giomer uses pre-reacted glass ionomer technology to form a stable phase of GIC in the restoration. The more extensive acid-base reaction and hydrogel layer of glass fillers are responsible for the high amount of release in Giomer on the first day. ${ }^{(19)}$

According to the results of the present study the comparison of the amount of initial fluoride release revealed that dental restoratives in descending order were RMGIC and Giomer. This order could be explained by the extent to which a glass ionomer matrix layer surrounds the glass filler in the set material. (20) It was reported that both the type and amount of resin used for the photochemical polymerization reaction may affect the fluoride release from RMGIC. ${ }^{(18)}$ Initial setting of RMGIC is performed by light-activated polymerization followed by an acid-base reaction that arises from the sorption of water. ${ }^{(21)}$

Moreover, the results of the present study revealed that the amount of fluoride released from both tested materials (RMGIC and Giomer) were significantly affected by the type of curing unit. The curing of RMGIC and Giomer with QTH showed a higher amount of fluoride release followed by LED and argon laser respectively. This could be explained by the light intensity of each curing unit as QTH has a lower intensity when compared to LED and argon laser which affected the degree of conversion. ${ }^{(22)}$ As the less release may result from the high degree of conversion from a double to a simple bond, which leads to the cohesion of polymer networks that reduces the mobility of ions such as fluoride. ${ }^{(23)}$

The high degree of conversion of a double bond to a single bond $(-\mathrm{c}=\mathrm{c}-)(\mathrm{c}-\mathrm{c})$. More polymerization resulted as the intensity increased by $400 \mathrm{Mw} /$ $\mathrm{cm}^{2}$. The increase in polymerization would result 
in entrapment of fluoride ions inside the lattice of the polymer. Finally, by increasing the intensity of the light cure, the amount of fluoride release will be decreased. ${ }^{(24)}$

In the present work, it was found that RMGIC and Giomer could be recharged and release fluoride slowly after exposure to fluoridated agents. This fact may be explained by the loosely bound water and the solutes in the porosities in the glass ionomer, which could be exchanged with an external medium by passive diffusion. ${ }^{(18)}$

In the present study, the total amount of fluoride re-released after recharging was compared among the materials and different curing methods. It was found that RMGIC showed significantly greater amounts than Giomer. Moreover, QTH showed a higher amount of fluoride re-release followed by LED and argon laser respectively. This is probably because GIC has well-established glass ionomer matrix around the glass filler particles. This phase could be responsible for fluoride release and recharging. ${ }^{(20)}$ This may allow deeper penetration of the fluoride recharging agent into materials having a substantial glass ionomer matrix component. Also, previous studies suggested that material with higher fluoride release has a higher fluoride recharging ability. ${ }^{(18,21)}$

\section{CONCLUSIONS}

- RMGIC has a higher fluoride release and recharging ability than Giomer.

- The use of QTH light-curing unit resulted in higher fluoride release/re-release when compared to LED and argon laser LCUs.

- The use of an LED light-curing unit resulted in higher fluoride release/re-release when compared to argon laser LCU.

- Both RMGIC and Giomer can release and recharge fluoride.

\section{REFERENCES}

1. Elshweekh RA, Bakry NS, Talaat DM, Ahmed DM. Fluoride release and rerelease after recharging of two hybrid resin restorations in primary teeth. A comparative in vitro study. Alex Dent J. 2019;44: 113-18.

2. Bayrak S, Tunc ES, Aksoy A, Ertas E, Guvenc D, Ozer S. Fluoride release and recharge from different materials used as fissure sealants. Eur J Dent. 2010; 4:245-50.

3. Westerman GH, Ellis RW, Latta MA, Powell GL. An in vitro study of enamel surface microhardness following argon laser irradiation and acidulated phosphate fluoride treatment. Pediatr Dent. 2003; 25:497-500.

4. Dionysopoulos D, Koliniotou-Koumpia E, HelvatzoglouAntoniades M, Kotsanos N. Fluoride release and recharge abilities of contemporary fluoride-containing restorative materials and dental adhesives. Dent Mater J. 2013; 32:296-304.

5. Wiegand A, Buchalla W, Attin T. Review on fluoride-releasing restorative materials-fluoride release and uptake characteristics, antibacterial activity and influence on caries formation. Dent Mater. 2007; 23: 343-62.

6. Francois P, Fouquet V, Attal JP, Dursun E. Commercially available fluoride-releasing restorative materials: A review and a proposal for classification. Materials (Basel). 2020; $13: 2313-41$.

7. Das UM, Prashanth ST. A comparative study to evaluate the effect of fluoride-releasing sealant cured by visible light, argon lasers, and light-emitting diode curing units: an in vitro study. J Indian Soc Pedod Prev Dent. 2009; 27:139-44.

8. Kouros P, Dionysopoulos D, Deligianni A, Strakas D, Sfeikos T, Tolidis K. Evaluation of photopolymerization efficacy and temperature rise of a composite resin using a blue diode laser (445 nm). Eur J Oral Sci. 2020;1: 1-7.

9. Eren D, Tutkan F. Investigation of the reliability of lightcuring units in Sivas City, Turkey. Niger J Clin Pract. 2019; 22:469-77.

10. Par M, Repusic I, Skenderovic H, Tarle Z. Wavelengthdependent light transmittance in resin composites: practical implications for curing units with different emission spectra. Clin Oral Investig. 2019; 23:4399-409.

11. El Zayat I, El Banna M, Ahmed DR, El Zayat A. Microleakage assessment and efficiency of two antimicrobial pit and fissure sealants: an in-vitro study. EDJ. 2017; 63: 711-19. 
12. Eldesouky HI, Hanno AG, Bakry NS, Ahmed DM. Marginal leakage evaluation of giomer and compomer in primary teeth (in-vitro study). Alex DJ. 2016; 14:188-93.

13. Makkai ZL, Szekely M, Fazakas Z, Nagy LM, Barta KM, Mathe BK. Fluoride release and uptake capability of glassionomer cements and compomers used as dental restorative materials. Mater Plast. 2019; 56: 548-51.

14. Das UM, Prashanth ST. A comparative study to evaluate the effect of fluoride releasing sealant cured by visible light, argon lasers, and light emitting diode curing units: an in vitro study. J Indian Soc Pedod Prev Dent. 2009; 27:139-44.

15. Suprastiwi E, Ayu Npa D. Analysis of fluoride released from GIC and RMGIC in saliva and dentino-enamel substance. Makara Seri Kesehatan. 2009; 13:53-8.

16. Bezerra NVF, Martins ML, de França Leite KL, de Medeiros MMD, de Almeida LD, Padilha WWN, et al. In Vitro evaluation of fluoride in saliva after topical application of professional use products. Pesqui. Bras. Odontopediatria Clín. Integr. 2019; 19:4005-8.

17. Yap AU, Tham SY, Zhu LY, Lee HK. Short-term fluoride release from various aesthetic restorative materials. Oper Dent. 2002; 27:259-65.

18. Bansal R, Bansal T. A Comparative Evaluation of the Amount of Fluoride Release and Re-Release after
Recharging from Aesthetic Restorative Materials: An in vitro Study. J Clin Diagn Res. 2015;9: 11-4.

19. Dhull KS, Nandlal B. Comparative evaluation of fluoride release from PRG Composites and compomer on application of topical fluoride: An in-vitro study. J Indian Soc Pedod Prev Dent. 2009; 27:27-32.

20. Itota T, Carrick TE, Yoshiyama M, McCabe JF. Fluoride release and recharge in giomer, compomer and resin composite. Dent Mater. 2004; 20:789-95.

21. Mousavinasab S M, Meyers I. Fluoride Release by Glass Ionomer Cements, Compomer and Giomer. Dent Res J. 2009; 6: 75-81.

22. Omidi BR, Gosili A, Jaber-Ansari M, Mahdkhah A. Intensity output and effectiveness of light curing units in dental offices. J Clin Exp Dent. 2018;10: 555-60.

23. Olmos-Olmos G, Teutle-Coyotecatl B, Román-Mendez $\mathrm{CD}$, Carrasco-Gutiérrez R, González-Torres M, ContrerasBulnes R, et al. The influence of light-curing time on fluoride release, surface topography, and bacterial adhesion in resin-modified glass ionomer cements: AFM and SEM in vitro study. Microsc Res Tech. 2021;1-10.

24. Sultan MA. The effect of light curing intensity on fluoride release from composite resin. Al-Rafidain Dent J. 2009; 9: $232-7$. 